\title{
EXISTENCE RESULTS FOR SYSTEMS OF ITERATIVE DIFFERENTIAL EQUATIONS
}

\author{
EVA BRESTOVANSKÁ, FRANTIŠEK JAROŠ, AND MILAN MEDVEĎ
}

Received 25 January, 2017

\begin{abstract}
In this article we give conditions for the existence of solutions of the initial value problem for systems of differential equations depending not only on the value of solutions but also on iterations of their coordinates.
\end{abstract}

2010 Mathematics Subject Classification: 34A12; 39B12; 47H09; 47H10

Keywords: initial value problem, iterative differential equations, non-expansive mappings, fixedpoint theorems

\section{INTRODUCTION}

Several works deal with first iterative initial value problems (see M.Fečkan [5], V.Berinde [2], S.Staněk [11-17]), while M.Medved' [8], S.Staněk [10] and P.Zhang and X.Gong [18] studied a functional differential equation of the more general form

$$
x^{\prime}(t)=F\left(t, x^{[1]}(t), x^{[2]}(t), \ldots, x^{[k]}(t)\right), \quad x(t) \in \mathbb{R} .
$$

Starting from results in [18] we prove an existence result for the following system of differential equations

$$
x^{\prime}(t)=f\left(t, x^{[1]}(t), x^{[2]}(t), \ldots, x^{[k]}(t)\right),
$$

with initial conditions

$$
x\left(t_{0}\right)=x_{0},
$$

where $x(t)=\left(x_{1}(t), x_{2}(t), \ldots, x_{n}(t)\right) \in \mathbb{R}^{n}, x^{[i]}(t)=\left(x_{1}^{[i]}(t), x_{2}^{[i]}(t), \ldots, x_{n}^{[i]}(t)\right)$, $x_{j}^{[i]}(t)$ is the $i$-th iteration of self-mapping $x_{j}(t), j=1,2, \ldots, n, f: \mathbb{R}^{n k+1} \rightarrow \mathbb{R}^{n}$ is continuous and, in addition, we show how one can approximate the non-unique solution of such kind of iterative differential equations by means of iterative techniques. The main idea is to use the powerful and more reliable technique of non-expansive

This work of the first and third author was supported by the Slovak Research and Development Agency under the contract No. APVV-14-0378 and by the Slovak Grant Agency VEGA No. 1/0078/17. 
operators and to adapt and use several convergence theorems from the theory of iterative approximation of fixed points of non-expansive mappings (see the monograph [1]).

\section{MAIN RESULTS}

In the proof of the main result we need the following lemma.

Lemma 1. Let

$\Phi_{M}=\left\{x \in C^{0}\left(I_{h}, \mathbb{R}^{n}\right):\|x(t)-x(s)\|=\max _{1 \leq j \leq n}\left|x_{j}(t)-x_{j}(s)\right| \leq M|t-s| ; t, s \in I_{h}\right\}$,

where $0<M<1$. If $g=\left(g_{1}, g_{2}, \ldots, g_{n}\right) \in \Phi_{M}$ and $g^{[i]}=\left(g_{1}^{[i]}, g_{2}^{[i]}, \ldots, g_{n}^{[i]}\right)$, where $g_{j}^{[i]}(t)$ the $i$-th iteration of $g_{j}(t)$ is for all $i \in\{1,2, \ldots, k\}, j \in\{1,2, \ldots, n\}$ well defined and the same for $h=\left(h_{1}, h_{2}, \ldots, h_{n}\right) \in \Phi_{M}$, then

$$
\left\|g^{[i]}-h^{[i]}\right\|_{I_{h}} \leq \frac{1-M^{i}}{1-M}\|g-h\|_{I_{h}}, \quad i=1,2, \ldots, k .
$$

Proof. Let $g, h \in \Phi_{M}, g=\left(g_{1}, g_{2}, \ldots, g_{n}\right), h=\left(h_{1}, h_{2}, \ldots, h_{n}\right)$. Since $\left|g_{j}(t)-g_{j}(s)\right| \leq\|g(t)-g(s)\| \leq M|t-s|$ and similarly $\left|h_{j}(t)-h_{j}(s)\right| \leq \| h(t)-$ $h(s) \| \leq M|t-s|$ for all $j \in\{1,2, \ldots, n\}$ and $t, s \in I_{h}$, by [18, Lemma 2.1], we have the inequality

This yields

$$
\left\|g_{j}^{[i]}-h_{j}^{[i]}\right\|_{I_{h}} \leq \frac{1-M^{i}}{1-M}\left\|g_{j}-h_{j}\right\|_{I_{h}} .
$$

$$
\begin{aligned}
\left\|g^{[i]}-h^{[i]}\right\|_{I_{h}} & =\max _{1 \leq j \leq n}\left\|g_{j}^{[i]}-h_{j}^{[i]}\right\|_{I_{h}} \\
& \leq \frac{1-M^{i}}{1-M} \max _{1 \leq j \leq n}\left\|g_{j}-h_{j}\right\|_{I_{h}}=\frac{1-M^{i}}{1-M}\|g-h\|_{I_{h}}
\end{aligned}
$$

for all $i \in\{1,2, \ldots, k\}$.

Theorem 1. Suppose that $f: \mathbb{R}^{n k+1} \rightarrow \mathbb{R}^{n}$ is a continuous mapping. Let there exists a positive constant $r$ such that

$$
(1-N) r>L_{0}=\max \left\{l_{1}^{0}, l_{2}^{0}, \ldots, l_{n}^{0}\right\},
$$

where $l_{j}^{0}=\left|x_{j}^{0}-t_{0}\right|, j=1,2, \ldots, n, x_{0}=\left(x_{1}^{0}, x_{2}^{0}, \ldots, x_{n}^{0}\right)$,

$$
N=\|f\|_{B\left(y_{0}, r\right)}<1,
$$

where $B\left(y_{0}, r\right)$ denotes the closed ball centered at $y_{0}=\left(t_{0}, x_{0}, \ldots, x_{0}\right)$ with radius $r$. Then the initial value problem (1.1), (1.2) has a solution defined on $\left[t_{0}-l, t_{0}+l\right]$ for any $l \in\left[\frac{L_{0}}{1-N}, r\right]$. 
Proof. The initial value problem (1.1), (1.2) is equivalent to the system of integral equations

$$
x_{j}(t)=x_{j}^{0}+\int_{t_{0}}^{t} f_{j}\left(s, x_{1}^{[1]}(s), \ldots, x_{n}^{[1]}(s), \ldots, x_{1}^{[k]}(s), \ldots, x_{n}^{[k]}(s)\right) \mathrm{d} s,
$$

where $j \in\{1,2, \ldots, n\}, \quad x\left(t_{0}\right)=x_{0}=\left(x_{1}^{0}, x_{2}^{0}, \ldots, x_{n}^{0}\right), \quad x(t)=\left(x_{1}(t), x_{2}(t), \ldots\right.$, $\left.x_{n}(t)\right), f=\left(f_{1}, f_{2}, \ldots, f_{n}\right)$. Let $l \in\left[\frac{L_{0}}{1-N}, r\right]$ and $t \in\left[t_{0}-l, t_{0}+l\right]$. Since $\left\|f_{j}\right\|_{B\left(y_{0}, r\right)}$ $\leq N=\|f\|_{B\left(y_{0}, r\right)}$, we obtain from (2.1) the following estimate for $x_{j}(t), j \in\{1,2, \ldots, n\}$ :

$$
\begin{aligned}
\left|x_{j}(t)-t_{0}\right| & \leq\left|x_{j}(t)-x_{j}\left(t_{0}\right)\right|+\left|x_{j}\left(t_{0}\right)-t_{0}\right| \leq N\left|t-t_{0}\right|+\left|x_{j}^{0}-t_{0}\right| \\
& \leq N l+l_{j}^{0} \leq N l+L_{0} \leq N l+l(1-N)=l .
\end{aligned}
$$

We have obtained the inequalities

$$
\left|x_{j}(t)-t_{0}\right| \leq l, \quad j=1,2, \ldots, n, t \in\left[t_{0}-l, t_{0}+l\right] .
$$

We will prove by induction the validity of the inequalities

$$
\left|x_{j}^{[i]}(t)-t_{0}\right| \leq l, \quad j=1,2, \ldots, n, i \in\{1,2, \ldots, k\}, t \in\left[t_{0}-l, t_{0}+l\right] .
$$

We assume that the inequalities (2.4) hold for all integer $i \in\{1,2, \ldots, k-1\}$. Then

$$
\begin{aligned}
\left|x_{j}^{[i+1]}(t)-t_{0}\right| & \leq\left|x_{j}^{[i+1]}(t)-x_{j}\left(t_{0}\right)\right|+\left|x_{j}\left(t_{0}\right)-t_{0}\right| \\
& =\left|x_{j}\left(x_{j}^{[i]}(t)\right)-x_{j}\left(t_{0}\right)\right|+\left|x_{j}^{0}-t_{0}\right| \leq N\left|x_{j}^{[i]}(t)-t_{0}\right|+l_{j}^{0} \\
& \leq N l+L_{0} \leq N l+l(1-N)=l .
\end{aligned}
$$

This means that the iterates $x_{j}^{[i]}$ are well defined on the interval $\left[t_{0}-l, t_{0}+l\right]$ for any $x=\left(x_{1}, x_{2}, \ldots, x_{n}\right) \in \Phi_{N}:=\left\{x \in C^{0}\left(I_{h}, \mathbb{R}^{n}\right): x_{j}\left(t_{0}\right)=x_{j}^{0}, j=1,2, \ldots, n ; \| x(t)-\right.$ $\left.x(s) \|=\max _{1 \leq j \leq n}\left|x_{j}(t)-x_{j}(s)\right| \leq N|t-s| ; t, s \in I_{h}\right\}$, and thus the operator

$$
\mathcal{E}: \Phi_{N} \rightarrow C^{0}\left(\left[t_{0}-l, t_{0}+l\right], \mathbb{R}^{n}\right), \quad \mathcal{E}=\left(\mathscr{E}_{1}, \mathcal{E}_{2}, \ldots, \mathcal{E}_{n}\right),
$$

where

$$
\mathscr{E}_{j} x(t)=x_{j}^{0}+\int_{t_{0}}^{t} f_{j}\left(s, x_{1}^{[1]}(s), \ldots, x_{n}^{[1]}(s), \ldots, x_{1}^{[k]}(s), \ldots, x_{n}^{[k]}(s)\right) \mathrm{d} s,
$$

and $j \in\{1,2, \ldots, n\}$ is well defined and obviously

$$
\mathscr{E}_{j} x\left(t_{0}\right)=x_{j}^{0},
$$


for any $x \in \Phi_{N}$ and $j \in\{1,2, \ldots, n\}$. Now we will prove that $\mathscr{E}$ is a self-mapping operator. We need to estimate

$$
\begin{aligned}
& \left\|\left(t, x^{[1]}(t), x^{[2]}(t), \ldots, x^{[k]}(t)\right)-\left(t_{0}, x_{0}, \ldots, x_{0}\right)\right\| \\
& =\max \left\{\left|t-t_{0}\right|,\left\|x^{[1]}(t)-x\left(t_{0}\right)\right\|, \ldots,\left\|x^{[k]}(t)-x\left(t_{0}\right)\right\|\right\} \\
& =\max \left\{\left|t-t_{0}\right|, \max _{1 \leq j \leq n}\left|x_{j}(t)-x_{j}\left(t_{0}\right)\right|, \max _{1 \leq j \leq n}\left|x_{j}\left(x_{j}^{[1]}(t)\right)-x_{j}\left(t_{0}\right)\right|,\right. \\
& \left.\ldots, \max _{1 \leq j \leq n}\left|x_{j}\left(x_{j}^{[k-1]}(t)\right)-x_{j}\left(t_{0}\right)\right|\right\} \\
& \leq \max \left\{\left|t-t_{0}\right|, N\left|t-t_{0}\right|, N \max _{1 \leq j \leq n}\left|x_{j}^{[1]}(t)-t_{0}\right|,\right. \\
& \left.\ldots, N \max _{1 \leq j \leq n}\left|x_{j}^{[k-1]}(t)-t_{0}\right|\right\} \\
& \leq \max \{l, N l, \ldots, N l\} \leq l \leq r, \quad t \in I_{l},
\end{aligned}
$$

i.e.

$$
\left(t, x^{[1]}(t), x^{[2]}(t), \ldots, x^{[k]}(t)\right) \in B\left(y_{0}, r\right) .
$$

This yields

$$
\begin{aligned}
& \left\|\mathscr{E} x\left(t_{2}\right)-\mathscr{E} x\left(t_{1}\right)\right\|=\max _{1 \leq j \leq n}\left|\mathscr{E}_{j} x\left(t_{2}\right)-\mathscr{E}_{j} x\left(t_{1}\right)\right| \\
& \leq \max _{1 \leq j \leq n}\left|\int_{t_{1}}^{t_{2}}\right| f_{j}\left(s, x_{1}^{[1]}(s), \ldots, x_{n}^{[1]}(s), \ldots, x_{1}^{[k]}(s), \ldots, x_{n}^{[k]}(s)\right)|\mathrm{d} s| \\
& \leq\|f\|_{B\left(y_{0}, r\right)}\left|\int_{t_{1}}^{t_{2}} \mathrm{~d} s\right| \leq N\left|t_{2}-t_{1}\right|
\end{aligned}
$$

for any $t_{1}, t_{2} \in I_{l}=\left[t_{0}-l, t_{0}+l\right]$. Thus (2.4) and (2.5) yield $\mathscr{G} \in \Phi_{N}$, i.e., $\mathcal{E}$ is self-mapping operator. For any $x, y \in \Phi_{N},\left(x\left(t_{0}\right)=x_{0}=y\left(t_{0}\right)\right)$ and any $t \in I_{l}$ we have

$$
\begin{aligned}
\|\mathscr{E} x(t)-\mathscr{E} y(t)\|= & \max _{1 \leq j \leq n}\left|\mathscr{E}_{j} x(t)-\mathscr{E}_{j} y(t)\right| \\
\leq & \max _{1 \leq j \leq n}\left|\int_{t_{0}}^{t}\right| f_{j}\left(s, x_{1}^{[1]}(s), \ldots, x_{n}^{[1]}(s), \ldots, x_{1}^{[k]}(s), \ldots, x_{n}^{[k]}(s)\right) \\
& -f_{j}\left(s, y_{1}^{[1]}(s), \ldots, y_{n}^{[1]}(s), \ldots, y_{1}^{[k]}(s), \ldots, y_{n}^{[k]}(s)\right)|\mathrm{d} s|
\end{aligned}
$$


By the Lemma 1,

$$
\begin{aligned}
\| & \left(s, x_{1}^{[1]}(s), \ldots, x_{n}^{[1]}(s), \ldots, x_{1}^{[k]}(s), \ldots, x_{n}^{[k]}(s)\right) \\
& -\left(s, y_{1}^{[1]}(s), \ldots, y_{n}^{[1]}(s), \ldots, y_{1}^{[k]}(s), \ldots, y_{n}^{[k]}(s)\right) \| \\
= & \max \left\{\left|x_{1}^{[1]}(s)-y_{1}^{[1]}(s)\right|, \ldots,\left|x_{n}^{[1]}(s)-y_{n}^{[1]}(s)\right|,\right. \\
& \left.\quad \ldots,\left|x_{1}^{[k]}(s)-y_{1}^{[k]}(s)\right|, \ldots,\left|x_{n}^{[k]}(s)-y_{n}^{[k]}(s)\right|\right\} \\
= & \max _{1 \leq i \leq k}\left\{\max _{1 \leq j \leq n}\left|x_{j}^{[i]}(s)-y_{j}^{[i]}(s)\right|\right\}=\max _{1 \leq i \leq k}\left\|x^{[i]}(s)-y^{[i]}(s)\right\| \\
\leq & \max _{1 \leq i \leq k}\left\|x^{[i]}-y^{[i]}\right\|_{I_{l}} \leq \max _{1 \leq i \leq k}\left\{\frac{1-N^{i}}{1-N}\|x-y\|_{I_{l}}\right\} \\
= & \frac{1-N^{k}}{1-N}\|x-y\|_{I_{l}}<\frac{1}{1-N}\|x-y\|_{I_{l}} .
\end{aligned}
$$

It follows from the uniform continuity of $f$ on the closed ball $B\left(y_{0}, r\right)$ that there for any $\varepsilon>0$ there exists $\delta(\varepsilon)>0$ such that the inequality

$$
\|\mathscr{E} x-\mathscr{E} y\|=\max _{1 \leq j \leq n}\left\|\mathscr{E}_{j} x-\mathscr{E}_{j} y\right\| \leq \varepsilon l
$$

holds for $\|x-y\|_{I_{l}}<\delta$ and therefore the operator $\mathcal{E}$ is continuous.

Since $\Phi_{N}$ is a convex compact subset of Banach space $C^{0}\left(I_{l}, \mathbb{R}^{n}\right)$ and $\mathscr{Y}$ is continuous operator, by the Schauder fixed point theorem we obtain that $\mathcal{E}$ has a fixed point $g_{0} \in \Phi_{N}$ and hence $g_{0}$ is a solution of the initial value problem for system of differential equations (1.1) associated with (1.2) on the interval $I_{l}=\left[t_{0}-l, t_{0}+l\right]$ and this completes the proof.

\subsection{Existence theorem and approximation of solutions}

The following result is a fixed point theorem for nonexpansive mappings (see e.g. in Berinde [1, Theorem 2.1]):

Lemma 2. Let $C$ be a nonempty closed convex and bounded subset of a uniformly convex Banach space B. Then any nonexpansive mapping $F: C \rightarrow C$ has a fixed point.

Definition 1. Let $C$ be a convex subset of a normed linear space $B$ and let $F: C \rightarrow$ $C$ be a self-mapping. Given any $x_{0} \in C$ and a real number $\lambda \in(0,1]$, the sequence $\left\{x_{n}\right\}$ defined by the formula

$$
x_{n+1}=(1-\lambda) x_{n}+\lambda F x_{n}, \quad n=0,1,2, \ldots
$$

is usually called the Krasnoselskij iteration, or Krasnoselskij-Mann iteration. 
Definition 2. Let $C$ be a convex subset of a normed linear space $B$ and let $F: C \rightarrow$ $C$ be a self-mapping. Given an $x_{0} \in C$ and a real numbers $\lambda_{n} \in[0,1]$, the sequence $\left\{x_{n}\right\}$ defined by the formula

$$
x_{n+1}=\left(1-\lambda_{n}\right) x_{n}+\lambda_{n} F x_{n}, \quad n=0,1,2, \ldots
$$

is usually called the Mann iteration.

It was shown by M.Krasnoselskij [7], in the case for $\lambda=1 / 2$, and later by H. Schaefer [9] for an arbitrary $\lambda \in(0,1)$, that if $B$ is a uniformly convex Banach space and $C$ is a convex and compact subset of $B$, then the M.Krasnoselskij iteration converges to a fixed point of $F$. M.Edelstein [4] proved that strict convexity of $B$ suffices for the same conclusion. The question if the assumption of the strict convexity of $B$ can be removed was answered affirmatively by the following S.Ishikawa [6] result:

Lemma 3. Let $C$ be a subset of a Banach space $B$ and let $F: C \rightarrow C$ be a nonexpansive mapping. For arbitrary $x_{0} \in C$, consider the Mann iteration process $\left\{x_{n}\right\}$ given by (2.9) under the following assumptions:

(a) $x_{n} \in C$ for all positive integers $n$;

(b) $0 \leq \lambda_{n} \leq b<1$ for all positive integers $n$;

(c) $\sum_{n=0}^{\infty} \lambda_{n}=\infty$.

If $\left\{x_{n}\right\}$ is bounded, then $x_{n}-F x_{n} \rightarrow 0$ as $n \rightarrow \infty$.

The following corollary of Lemma 3 will be particularly important for the second main result of our paper.

Corollary 1 (see in C.Chidume [3, Theorem 6.17]). Let $C$ be a convex and compact subset of a Banach space $B$ and let $F: C \rightarrow C$ be a nonexpansive mapping. If the Mann iteration process $\left\{x_{n}\right\}$ given by (2.9) satisfies the assumptions (a)-(c) of Lemma 3, then $\left\{x_{n}\right\}$ converges strongly to a fixed point of $F$.

We consider the initial value problem

(1) $x^{\prime}(t)=f\left(t, x^{[1]}(t), x^{[2]}(t), \ldots, x^{[k]}(t)\right), \quad t \in[a, b]$

(2) $x\left(t_{0}\right)=x_{0}$.

where $t_{0} \in[a, b], x_{0}=\left(x_{1}^{0}, x_{2}^{0}, \ldots, x_{n}^{0}\right) \in(a, b)^{n k}, f \in C^{0}\left([a, b]^{n k+1}, \mathbb{R}^{n}\right)$ are given and $[a, b]^{n k+1}$ denotes the product $n k+1$ intervals $[a, b]$. Let us put

$$
A_{t}=\max \{t-a, b-t\}, \quad B_{x}^{j}=\min \left\{x_{j}-a, b-x_{j}\right\} .
$$

We are interested here in solution from the space $C^{1}\left([a, b],[a, b]^{n}\right)$ belonging to the class

$$
\begin{aligned}
\Phi_{K}:= & \left\{x \in C^{0}\left([a, b],[a, b]^{n}\right): x_{j}\left(t_{0}\right)=x_{j}^{0}, j=1,2, \ldots, n ;\right. \\
& \left.\|x(t)-x(s)\|=\max _{1 \leq j \leq n}\left|x_{j}(t)-x_{j}(s)\right| \leq K|t-s| ; t, s \in[a, b]\right\},
\end{aligned}
$$

where $K=\|f\|_{[a, b]^{n k+1}}$. 
Theorem 2. Assume that the following conditions are satisfied for the initial value problem (1.1), (1.2):

(i) $f: \mathbb{R}^{n k+1} \rightarrow \mathbb{R}^{n}$ is continuous and $[a, b]$ is a compact interval containing $t_{0}$, $x_{j}^{0}, j=1,2, \ldots, n$;

(ii) there exist $L>0$ such that $\|f(t, u)-f(t, v)\| \leq L\|u-v\|$ for all $t \in[a, b]$ and $u, v \in[a, b]^{n k}$;

(iii) let $K=\|f\|_{[a, b]^{n k+1}}>0$ and let one of the following conditions hold:

(a) $K A_{t_{0}} \leq B_{x_{0}}^{j}$, for all $j=1,2, \ldots, n$, where $A_{t_{0}}, B_{x_{0}}^{j}$ are defined by $(2.10)$

(b) $t_{0}=a, K(b-a) \leq b-x_{j}^{0}, j=1,2, \ldots, n, f(t, u) \geq 0$ for all $(t, u) \in$ $[a, b]^{n k+1}$

(c) $t_{0}=b, K(b-a) \leq x_{j}^{0}-a, j=1,2, \ldots, n, f(t, u) \geq 0$ for all $(t, u) \in$ $[a, b]^{n k+1}$.

In addition

(iv) (A) $L A_{t_{0}} \frac{1-K^{k}}{1-K} \leq 1$, if $K<1$,

(B) $L A_{t_{0}} k \leq 1$, if $K=1$.

Then there exists at least one solution $x^{*}$ of problem (1.1), (1.2) in the class $\Phi_{K}$, which can be approximated by the Mann iteration

$$
x_{m+1}=\left(1-\lambda_{m}\right) x_{m}+\lambda_{m} \mathscr{E} x_{m}, \quad m=0,1,2, \ldots,
$$

where $\left\{\lambda_{m}\right\}$ is a given sequence of real numbers satisfying the assumptions (b)-(c) formulated in Lemma 3 and the operator

$$
\mathscr{E}: \Phi_{K} \rightarrow C^{0}\left([a, b], \mathbb{R}^{n}\right), \quad \mathcal{E}=\left(\mathscr{E}_{1}, \mathscr{E}_{2}, \ldots, \mathscr{E}_{n}\right)
$$

is defined by

$$
\mathscr{E}_{j} x(t)=x_{j}^{0}+\int_{t_{0}}^{t} f_{j}\left(s, x_{1}^{[1]}(s), \ldots, x_{n}^{[1]}(s), \ldots, x_{1}^{[k]}(s), \ldots, x_{n}^{[k]}(s)\right) \mathrm{d} s,
$$

where $j \in\{1,2, \ldots, n\}$.

Proof. $\Phi_{K}$ is nonempty convex compact subset of Banach space

$\left(C^{0}\left([a, b],[a, b]^{n}\right)\right)$ with usual supremum norm. It is clear that $x^{*} \in \Phi_{K}$ is a solution of the initial value problem (1.1), (1.2) if and only if $x^{*}$ is a fixed point of $\mathcal{E}$, i.e., $x^{*}=\mathscr{E} x^{*}$. Our aim is to shaw that $\mathscr{E}$ has a fixed point in $\Phi_{K}$. We will apply Schauder fixed point theorem or Banach fixed point theorem.

First we prove that $\Phi_{K}$ is a invariant set with respect to $\mathscr{E}$, i.e., $\mathscr{E}\left(\Phi_{K}\right) \subseteq \Phi_{K}$. 
If the condition (iii)(a) holds, then for any $x \in \Phi_{K}, t \in[a, b]$ and for all $j \in$ $\{1,2, \ldots, n\}$ we have

$$
\begin{aligned}
\mathscr{E}_{j} x(t) & =x_{j}^{0}+\int_{t_{0}}^{t} f_{j}\left(s, x_{1}^{[1]}(s), \ldots, x_{n}^{[1]}(s), \ldots, x_{1}^{[k]}(s), \ldots, x_{n}^{[k]}(s)\right) \mathrm{d} s \\
& \leq x_{j}^{0}+\left|\int_{t_{0}}^{t} f_{j}\left(s, x_{1}^{[1]}(s), \ldots, x_{n}^{[1]}(s), \ldots, x_{1}^{[k]}(s), \ldots, x_{n}^{[k]}(s)\right) \mathrm{d} s\right| \\
& \leq x_{j}^{0}+K\left|t-t_{0}\right| \leq x_{j}^{0}+K A_{t_{0}} \leq x_{j}^{0}+B_{x_{0}}^{j} \leq x_{j}^{0}+\left(b-x_{j}^{0}\right)=b
\end{aligned}
$$

and

$$
\begin{aligned}
\mathscr{E}_{j} x(t) & \geq x_{j}^{0}-\left|\int_{t_{0}}^{t} f_{j}\left(s, x_{1}^{[1]}(s), \ldots, x_{n}^{[1]}(s), \ldots, x_{1}^{[k]}(s), \ldots, x_{n}^{[k]}(s)\right) \mathrm{d} s\right| \\
& \geq x_{j}^{0}-K\left|t-t_{0}\right| \geq x_{j}^{0}-K A_{t_{0}} \geq x_{j}^{0}-B_{x_{0}}^{j} \geq x_{j}^{0}-\left(x_{j}^{0}-a\right)=a .
\end{aligned}
$$

The cases (iii)(b) and (iii)(c) can be treated in a similar way and so we have that for any $x \in \Phi_{K}$ and $t \in[a, b]$, one has $\mathscr{\xi}_{j} x(t) \in[a, b]$ for all $j \in\{1,2, \ldots, n\}$.

Now, for any $t_{1}, t_{2} \in[a, b]$ we have

$$
\begin{aligned}
& \left\|\mathscr{E x}\left(t_{2}\right)-\mathscr{E} x\left(t_{1}\right)\right\|=\max _{1 \leq j \leq n}\left|\mathscr{E}_{j} x\left(t_{2}\right)-\mathscr{E}_{j} x\left(t_{1}\right)\right| \\
& \leq \max _{1 \leq j \leq n}\left|\int_{t_{1}}^{t_{2}}\right| f_{j}\left(s, x_{1}^{[1]}(s), \ldots, x_{n}^{[1]}(s), \ldots, x_{1}^{[k]}(s), \ldots, x_{n}^{[k]}(s)\right)|\mathrm{d} s| \\
& \leq\|f\|_{[a, b]^{n k+1}}\left|\int_{t_{1}}^{t_{2}} \mathrm{~d} s\right| \leq K\left|t_{2}-t_{1}\right| .
\end{aligned}
$$

By the formula (2.6) we have $\mathscr{E}_{j} x\left(t_{0}\right)=x_{j}^{0}$ for any $x \in \Phi_{K}$ and $j \in\{1,2, \ldots, n\}$. Therefore $\mathscr{E}: \Phi_{K} \rightarrow \Phi_{K}$ (i.e., $\mathcal{E}$ is a selfmapping of $\Phi_{K}$ ).

From the assumptions ( $i$ i i $)$ and the definitions of $A_{t_{0}}$ and $B_{x_{0}}^{j}$, it follows that $K \leq$ 1. Therefore according to Lemma 1 for any $x, y \in \Phi_{K}, s \in[a, b], i \in\{2,3, \ldots, k\}$, $j \in\{1,2, \ldots, n\}$ and $K<1$ the following holds:

$$
\begin{aligned}
& \left|x_{j}^{[i]}(s)-y_{j}^{[i]}(s)\right| \\
& \leq\left|x_{j}\left(x_{j}^{[i-1]}(s)\right)-x_{j}\left(y_{j}^{[i-1]}(s)\right)\right|+\left|x_{j}\left(y_{j}^{[i-1]}(s)\right)-y_{j}\left(y_{j}^{[i-1]}(s)\right)\right| \\
& \left.\leq K \mid x_{j}^{[i-1]}(s)-y_{j}^{[i-1]}(s)\right)\left|+\max _{\xi \in[a, b]}\right| x_{j}(\xi)-y_{j}(\xi) \mid \\
& \left.\leq K\left(K \mid x_{j}^{[i-2]}(s)-y_{j}^{[i-2]}(s)\right)\left|+\max _{\xi \in[a, b]}\right| x_{j}(\xi)-y_{j}(\xi) \mid\right)+\max _{\xi \in[a, b]}\left|x_{j}(\xi)-y_{j}(\xi)\right| \\
& \leq \cdots \leq\left(K^{i-1}+K^{i-2}+\cdots+1\right) \max _{\xi \in[a, b]}\left|x_{j}(\xi)-y_{j}(\xi)\right| \leq \frac{1-K^{k}}{1-K}\left\|x_{j}-y_{j}\right\|,
\end{aligned}
$$


and if $K=1$ then

$$
\left|x_{j}^{[i]}(s)-y_{j}^{[i]}(s)\right| \leq k\left\|x_{j}-y_{j}\right\| .
$$

Now if we take any $x, y \in \Phi_{K}$ and any $t \in[a, b]$, for $K<1$ we have

$$
\begin{aligned}
& \|\mathscr{E} x(t)-\mathscr{E} y(t)\|=\max _{1 \leq j \leq n}\left|\mathscr{E}_{j} x(t)-\mathscr{E}_{j} y(t)\right| \\
& \leq \max _{1 \leq j \leq n}\left|\int_{t_{0}}^{t}\right| f_{j}\left(s, x_{1}^{[1]}(s), \ldots, x_{n}^{[1]}(s), \ldots, x_{1}^{[k]}(s), \ldots, x_{n}^{[k]}(s)\right) \\
& -f_{j}\left(s, y_{1}^{[1]}(s), \ldots, y_{n}^{[1]}(s), \ldots, y_{1}^{[k]}(s), \ldots, y_{n}^{[k]}(s)\right)|\mathrm{d} s| \\
& \leq \mid \int_{t_{0}}^{t} L \|\left(x_{1}^{[1]}(s), \ldots, x_{n}^{[1]}(s), \ldots, x_{1}^{[k]}(s), \ldots, x_{n}^{[k]}(s)\right) \\
& -\left(y_{1}^{[1]}(s), \ldots, y_{n}^{[1]}(s), \ldots, y_{1}^{[k]}(s), \ldots, y_{n}^{[k]}(s)\right) \| \mathrm{d} s \mid \\
& \leq L\left|\int_{t_{0}}^{t} \max _{1 \leq i \leq k}\left\{\max _{1 \leq j \leq n}\left|x_{j}^{[i]}(s)-y_{j}^{[i]}(s)\right|\right\} \mathrm{d} s\right| \\
& \leq L\left|\int_{t_{0}}^{t} \max _{1 \leq j \leq n}\left\{\frac{1-K^{k}}{1-K}\left\|x_{j}-y_{j}\right\|\right\} \mathrm{d} s\right| \\
& \leq L \frac{1-K^{k}}{1-K}\|x-y\|\left|t-t_{0}\right| \leq L A_{t_{0}} \frac{1-K^{k}}{1-K}\|x-y\| .
\end{aligned}
$$

(In the case $K=1$ we have $\|\mathscr{E} x(t)-\mathscr{E} y(t)\| \leq L A_{t_{0}} k\|x-y\|$ ).

Now, by taking the supremum in the last inequalities, we get

$$
\|\mathscr{E} x-\mathscr{E} y\| \leq L A_{t_{0}} \frac{1-K^{k}}{1-K}\|x-y\|,\left(\|\mathcal{E} x-\mathscr{E} y\| \leq L A_{t_{0}} k\|x-y\|\right)
$$

and so from the conditions (iv) it follows that operator $\mathcal{G}$ is nonexpansive and hence continuous. Now it remains to apply the Schauder fixed point theorem to obtain the first part theorem (if $L A_{t_{0}} \frac{1-K^{k}}{1-K}<1$ for $K<1$, or $L A_{t_{0}} k<1$ for $K=1$, then $\mathcal{G}$ is a contraction mapping and hence in view Banach fixed point theorem, has a unique solution) and Corollary 1 to get the second one. This completes the proof.

\section{REFERENCES}

[1] V. Berinde, Iterative approximation of fixed points. 2nd revised and enlarged ed., 2nd ed. Berlin Heidelberg: Springer-Verlag, 2007, vol. 1912, doi: 10.1007/978-3-540-72234-2.

[2] V. Berinde, "Existence and approximation of solutions of some first order iterative differential equations." Miskolc Math. Notes, vol. 11, no. 1, pp. 13-26, 2010.

[3] C. Chidume, Geometric properties of Banach spaces and nonlinear iterations. Berlin: Springer, 2009. doi: 10.1007/978-1-84882-190-3.

[4] M. Edelstein , “A remark on a theorem of M. A. Krasnoselskij,” Amer. Math. Monthly, vol. 73, pp. 509-510, 1966, doi: 10.2307/2315474. 
[5] M. Fečkan, "On a certain type of functional differential equations." Math. Slovaca, vol. 43, no. 1, pp. 39-43, 1993.

[6] S. Ishikawa, "Fixed points and iteration of a nonexpansive mapping in a Banach space." Proc. Amer. Math. Soc., vol. 59, pp. 65-71, 1976, doi: 10.2307/2042038.

[7] M. Krasnosel'skij, "Zwei Bemerkungen über die Methode der sukzessiven Approximationen." Uspekhi Mat. Nauk, vol. 10, no. 1(63), pp. 123-127, 1955.

[8] M. Medved̆, "On minimal periods of functional-differential equations and difference inclusions." Ann. Polon. Math., vol. 54, no. 3, pp. 263-270, 1991.

[9] H. Schaefer, "Über die Methode sukzessiver Approximationen." Jahresber. Deutsch. Math.Verein., vol. 59, pp. 131-140, 1957.

[10] S. Staněk, "Global properties of solutions of iterative-differential equations." Funct. Differ. Equ., vol. 5, no. 3-4, pp. 463-481, 1998.

[11] S. Staněk, "Global properties of solutions of the functional differential equation $X(T) X^{\prime}(T)=$ $K X(X(T)), 0<|K|<1$." Funct. Differ. Equ., vol. 9, no. 3-4, pp. 527-550, 2002.

[12] S. Staněk, "Properties of maximal solutions of functional-differential equations with statedependent deviations." Funct. Differ. Equ., vol. 16, no. 4, pp. 729-749, 2009.

[13] S. Staněk, "On global properties of solutions of functional differential equation $x^{\prime}(t)=x(x(t))+$ x(t).” Dynam. Systems. Appl., vol. 4, no. 2, pp. 263-278, 1995.

[14] S. Staněk, "Global properties of decreasing solutions of the equation $X^{\prime}(T)=X(X(T))+X(T)$." Funct. Differ. Equ., vol. 4, no. 1-2, pp. 191-213, 1997.

[15] S. Staněk, "Global properties of decreasing solutions for the equation $x^{\prime}(t)=x(x(t))-b x(t), b \in$ $(0,1)$." Soochow J. Math., vol. 26, no. 2, pp. 123-134, 2000.

[16] S. Staněk, "Global properties of increasing solutions to the equation $x^{\prime}(t)=x(x(t))-b x(t)$, $b \in(0,1)$." Soochow J. Math., vol. 26, no. 1, pp. 37-65, 2000.

[17] S. Staněk, "On global properties of solutions of the equation $y^{\prime}(t)=a y(t-b y(t))$." Hokkaido Math. J., vol. 30, no. 1, pp. 75-89, 2001.

[18] P. Zhang and X. Gong, "Existence of solutions for iterative differential equations." Electron. J. Differential Equations, vol. 2014, p. 10, 2014.

\section{Authors' addresses}

\section{Eva Brestovanská}

Comenius University, Faculty of Management, Department of Economics and Finance, Odbojárov St., 83104 Bratislava, Slovakia

E-mail address: Eva.Brestovanska@fm.uniba.sk

František Jaroš

Comenius University, Faculty of Mathematics, Physics and Informatics, Department of Mathematical Analysis and Numerical Mathematics, 84248 Bratislava, Slovakia

E-mail address: Frantisek.Jaros@fmph.uniba.sk

\section{Milan Medved}

Comenius University, Faculty of Mathematics, Physics and Informatics, Department of Mathematical Analysis and Numerical Mathematics, 84248 Bratislava, Slovakia

E-mail address: Milan.Medvedefmph.uniba.sk 